\title{
PHOTODYNAMIC EFFECTS OF VITAL STAINING WITH DIAZINE GREEN (JANUS GREEN) ON LIVING BULL SPERMATOZOA ${ }^{1}$
}

\author{
C. VAN D U I J N, Jr. \\ Biophysics Department, "Schoonoord", Research Institute for Animal Husbandry, \\ Utrecht, The Netherlands \\ Received February 24, 1961
}

Diazinle green or Janus green has been known for a long time as a specific vital stain for mitochondria $[1,8,9,15]$. It has been stated to be extremely toxic, however, killing cells in tissue culture in a few hours, even in concentrations as low as $1: 200,000[8]$.

Recent investigations with regard to the effects of acridine orange on cells in tissue culture $[6,7]$ and on spermatoxoa $[2,3,4]$ demonstrated that the observed adverse effects in low concentrations can be accounted for completely as being due to optical sensitization. Since every adsorbed dye may, in principle, be capable of sensitizing its substrate, a comparative study of some further dyes and fluorochromes was started [4], since exact knowledge of toxic effects of such substances on spermatozoa is important with respect to their use for evaluation of semen quality $[4,16]$. Among vital stains diazine green is of special interest owing to its specific adsorption to the mitochondria, which in sperm have to provide the energy for locomotion, so that any interference with mitochondrial activity may be expected to have a definite effect on swimming velocity, which can be accurately measured with photo-electric methods [12, 14].

\section{MATERIALS AND METHODS}

Semen handling.- Freshly ejaculated semen from Dutch-Friesian and red-andwhite Meuse-Rhine-Yssel bulls was allowed to cool to $29 \pm 0.5^{\circ} \mathrm{C}$ and then diluted ten times with standardized ultramicroscopically clear egg-yolk-citrate buffer [11] of $30^{\circ} \mathrm{C}$. After this first dilution the samples were slowly cooled to $\sim 4^{\circ} \mathrm{C}$ by placing the tubes in a larger glass beaker, which in its turn was placed in a Dewar vessel with ice. About an hour later further dilutions of seven times were made, every sample having been split into a number of portions. For determining the effect of

3 138th Publication of the "Schoonoord" Research Institute for Animal Husbandry, Hoogt 10, Utrecht, the Netherlands. 
concentration, the split portions were diluted with the same diluent to which different amounts of diazine ${ }^{\circledR}$ green (Merck, pro analysi) had been added. For determining the effect of illumination, after the first dilution each sample was split into four portions, to two of which diazine green in a final concentration of 20 p.p.m. $(1: 50,000)$ was added.

The medium to be used in the second dilution (either with or without diazine green) was poured gently on top of the fluid to avoid mixing, and the sample was allowed to stand in the Dewar vessel for 20 min, in which period a slow and gradual preliminary mixing by diffusion takes place. Then complete mixing was accomplished by gentle turning. This cautious procedure is required to avoid shocking the spermatozoa when semen is diluted.

Two portions, one with and one without dye addition, were kept in darkness, in a Dewar vessel with ice, the tubes being isolated from direct contact with the ice by an air mantle. The tubes used for irradiation (also isolated by an air mantle) were placed in a glass beaker with ice and water, separated from the light source (a $150 \mathrm{~W}$, $220 \mathrm{~V}$ incandescent frosted lamp with an internal silver mirror) by a cooling cylinder filled with water and ice. At regular intervals drops were taken from the test tubes and brought into the photo-electric equipment.

Diluent. - This consisted of a 15 per cent egg-yolk-containing sodium citrate buffer, adjusted to $\mathrm{pH} 6.75 \pm 0.05$ measured at $22-23^{\circ} \mathrm{C}$, corresponding to $6.80 \pm 0.05$ (McInnes scale) at $37^{\circ} \mathrm{C}$, i.e. completely neutral, since the neutral point in the ionic equilibrium of water at $37^{\circ} \mathrm{C}$ is $\mathrm{pH} 6.81$.

The buffer contained $500 \mu \mathrm{g}$ streptomycin and 1000 I.U. penicillin per $\mathrm{ml}$ to prevent bacterial growth on incubation. Freezing point depression $\Delta=-0.54 \pm$ $0.02^{\circ} \mathrm{C}$; viscosity $\eta=1.04 \pm 0.02 \mathrm{cp}$ at $37.1^{\circ} \mathrm{C}$; refractive index $n_{D}-1.3456$ at $40^{\circ} \mathrm{C}$; density $d-1.017$ at $20^{\circ} \mathrm{C}$; buffer capacity $d A / d p H=0.03$. The buffer was made ultramicroscopically clear by ultracentrifugation, followed by ultrafiltration [11]. To prevent coagulation of the egg-yolk as well as changing of the physico-chemical constants, the addition of the dye had to be done in a special way. An aqueous solution of diazine green was made and the required amounts were pipetted into small test tubes. These were then placed in an incubator and left to evaporate the water. Each tube now contained an exact amount of dry dye, which was dissolved in the egg-yolk-citrate buffer. If a slight turbidity should occur, the final medium was filtered prior to use, which did not alter its physico-chemical constants to any measurable extent.

Velocily measurements.-These were performed with the photo-electric apparatus developed in our Institute by Rikmenspoel $[12,14]$.

By means of a leukocyte pipette drops of the diluted semen $(13.5 \pm 0.3 \mu \mathrm{l})$ were brought into a microchamber, produced by sandwiching a plastic foil (Clarifoil $\otimes$ ) with a central hole punched into it, between two slides of dark field quality, held firmly together by a metal frame [12]. The microchamber with the specimens was warmed up for 5 min to $37^{\circ} \mathrm{C}$. Then the dark field image of individual swimming spermatozoa was projected on to a small aperture "viewed" by a photomultiplier through an auxiliary lens. Every time a spermatozoön passes by the area "scen" by the photomultiplier the latter receives light impulses and emits a corresponding number of photo-electrons. The resulting fluctuating photocurrent is amplified and recorded. 
The length of the recorded track of each specimen is a measure of its velocity. The accuracy of this measurement for one single specimen is \pm 8 per cent, the main part of this not being due to measuring error, but to statistical variation of sperm size [12]. Of course, the error in the mean velocity of a sample is considerably smaller, dependent on the number of specimens measured; on the average it amounts to 2--3 per cent for one recording.

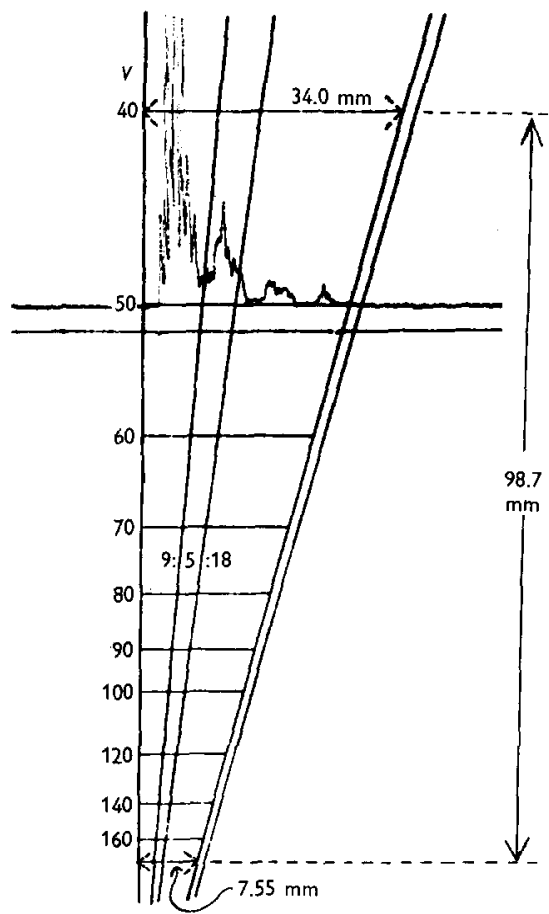

Fig. 1.

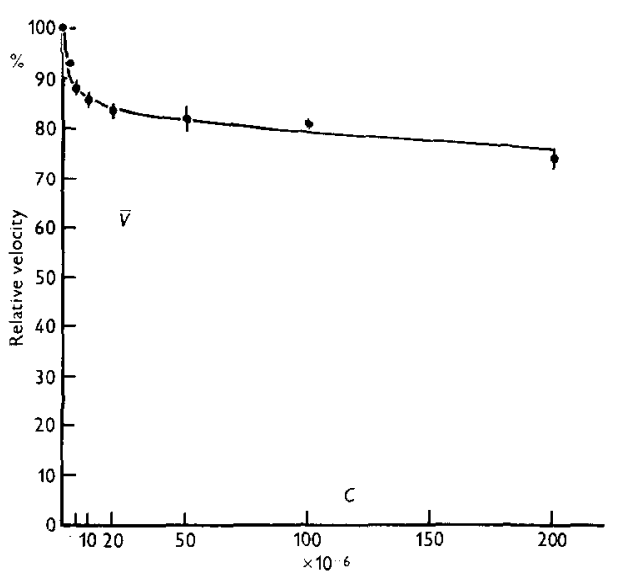

Fig. 2.

Fig. 1.-Example of recording of sperm and measuring scale. The nomograph scales are engraved in a rectangular piece of polymethylmethacrylate ("Perspex" $B$ ). The vertical axis is brought to coincide with the entry of the sperm head at the recording paper and then the device is shifted along this axis till the subdivisions of head, mid-piece and (bent) tail coincide with the three sloping lines respectively. The fourth sloping line indicates the correction to be added to the sperm length if the specimen has passed by at the border of the aperture. The speed of the recorded specimen is $57 \mu / \mathrm{sec}$; it is shown at the mid-mark of the velocity class $40-60$.

Fig. 2.-Mean velocity of bull spermatozoa as a function of the concentration of diazine green at constant dosage of illumination. Pooled data of four ejaculates from four different bulls, each measured twice at about $24 \mathrm{hr}$ interval.

The "Mingograf 42" manufactured by Messrs Elema Järnh, Stockholm, Sweden, was used for taking the recordings, at a speed of $2.5 \mathrm{~cm} / \mathrm{sec}$. In order to keep light effects during recording constant, all recordings were extended over a period of $8 \mathrm{~min}$, which was the time required to get a sufficient number of specimens recorded at high dye concentrations or prolonged illumination prior to recording. From the 
recordings the numbers of normally moving spermatozoa and their swimming velocities were determined. The velocities of individual specimens were determined by means of the nomograph I designed previously [5] (Fig. 1).

Obviously, spermatozoa moving slowly have a smaller chance of passing by the area "viewed" by the photomultiplier than fast ones. This is accounted for in the calculation of the mean velocity by dividing the number of sperms counted in each class by the class mid-marks to get the true $f(v)$ values, the probabilities of sperms passing by the specific spot being proportional to their individual velocities. The mean velocity then follows from $\bar{v}=\Sigma n / \Sigma f(v)$. The mean velocities under standardized conditions without addition of dyes or other special substances are of the order of $100 \mu / \mathrm{sec}[5,12,13]$.

The average number $(N)$ per unit volume, of spermatozoa swimming normally is related to the mean velocity $\bar{v}$, and the number of passages per unit of time $n$, according to:

$$
N=\frac{n}{\bar{v}} \cdot \frac{1}{Q d}
$$

where $Q$ is a constant depending on the diameter of the aperture passed by the projected image of the individual sperms and on the characteristics of the movement of the head during passage [12,14]. Its numerical value is $15.5 \pm 1.2 \mu$; $d$ is the thickness of the liquid film in the microchamber, which in the present investigations was $40 \mu$. According to eq. (1) the average number of spermatozoa swimming normally is proportional to the ratio $n / \bar{v}$ and this ratio, expressed in arbitrary units, may be used for comparative purposes. Its average confidence limit is about 10 per cent for one recording.

\section{RESULTS}

\section{Experiments with Varying Dye Concentration at Constant Dosage of Illumination}

The concentrations used ranged from 2-200 p.p.m. At each concentration two recordings were made: the first directly following mixing of the semen with the dye-containing diluent and the second one about $24 \mathrm{~h}$ later, the semen with dye addition having been stored in the dark in a Dewar vessel with icc, as described under "Methods". In this way it was possible to detect whether there would be a toxic effect in absence of light. If there should be a toxic effect in darkness, the rate of velocity decrease with time should be greater for the samples with dye addition than for the controls. No such difference was found, the rates of velocity decrease with time, $-\partial \bar{v} / \partial t$, being $0.72 \pm 0.06(S . E).(\mu / \mathrm{sec}) / \mathrm{h}(n=27)$ for the samples with diazine green and $0.71 \pm 0.13(S . E).(\mu / \mathrm{sec}) / \mathrm{h}(n=9)$ for the controls without dye addition.

The curves representing the relationship between the mean velocity and 
the dye concentration obtained from the direct recordings and from those obtained after about $24 \mathrm{hr}$ run parallel. 'This means that they may be normalized by shifting to the same initial value, and then pooled together. Fig. 2 gives the resultant curve obtained by pooling the data of four ejaculates

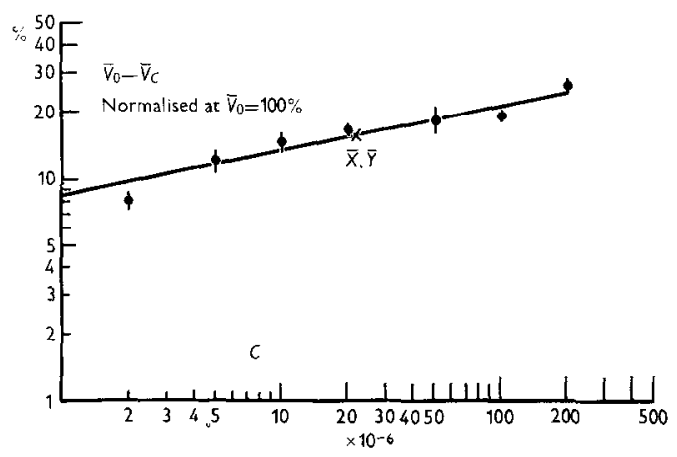

Fïg. 3 a.

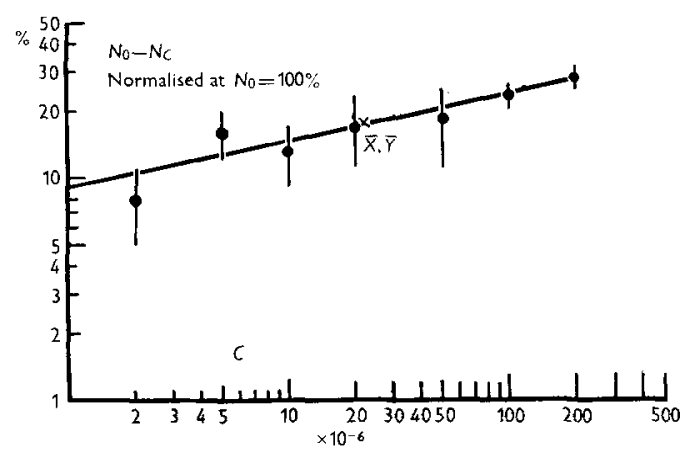

Fig. $3 \mathrm{~b}$.

Fig. 3.-Same data as represented in Fig. 2, after a straight-line transformation. Log-log plot of $\bar{v}_{0}-\bar{r}_{c}$ vs. $C$, and of $N_{0}-N_{c}$ vs. $C$. Transformation according to equations (2) and (3), following the Freundlich adsorption isotherm.

from four different bulls. This curve can be accurately described by the equation:

$$
\bar{v}_{c}=\bar{v}_{0}-k C^{(p / q)}
$$

where $\bar{v}_{\mathrm{c}}=$ mean velocity at concentration $C, \bar{v}_{0}=$ mean velocity without dye addition, $k, p, q$ are empirical constants, with $(p / q) \neq 1$ and $p<q$.

Of course the exponent $(p / q)$ can be substituted by a single symbol; writing it as a quotient identifies it immediately as essentially a broken exponent. A further reason for preferring this notation is given later on. 
This equation yields a straight line if $\log \left(\bar{v}_{0}-\bar{v}_{c}\right)$ is plotted as a function of $\log C$ (Fig. 3). On this plot, $\log k$ is found at the intersection of the curve with an ordinate at $\log C=0$, whereas $(p / q)$ is the slope constant of the curve; in the present investigations its numerical value was of the order of $0.1-0.2$.

For the number of spermatozoa moving normally the same relationship was found:

$$
N_{c}=N_{0}-k C^{(p / q)}
$$

The constants of the curves obtained for $\bar{v}$ and $N$ were statistically not different: $P\left\{b_{v}-b_{N}\right\}=0.95$.

Equations (2) and (3) represent the Freundlich adsorption isotherm, if it is assumed that the effect of the adsorbed dye is strictly proportional to the mass $M_{A}$ adsorbed per unit of cell area engaged in the adsorption process and functionally affected by it. The exponent $(p / q)$ then represents the product of the Freundlich exponent $1 / q$ with a proportionality constant $p$, which may or may not be dependent on other conditions such as the illumination level. The Freundlich equation is empirical; therefore it was also checked whether the data would fit the Langmuir equation (4), which is theoretically founded.

$$
M_{A}=\frac{k C}{C+a},
$$

where $k$ and $a$ are constants, the first one representing the specific adsorptive power of the adsorbing surface with respect to the adsorbed substance (dimension: mass per area), whereas $a$ has the dimension of a concentration

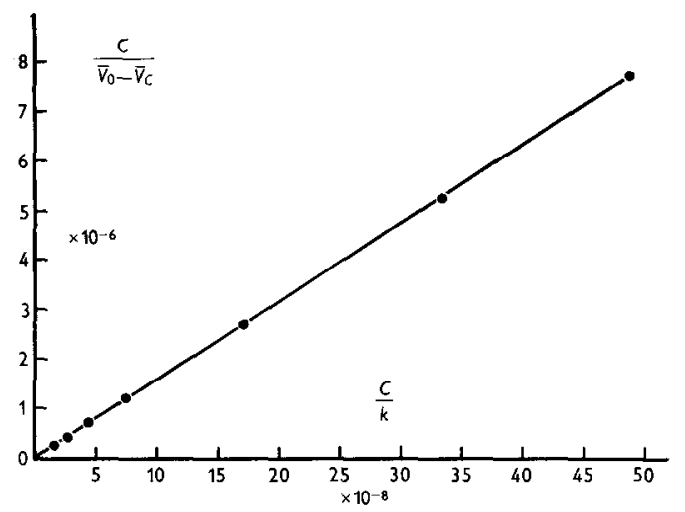

lig. 4. Same data as represented in Fig. 2, after a straight-line transformation according to equations (5) and (6), in agreement with the Langmuir adsorption isotherm. 
(mass per volume). Assuming that $\left(\bar{v}_{0}-\bar{v}_{c}\right)$ is proportional to the adsorbed mass per unit of affected area $\left(M_{A}\right)$, we get:

$$
\bar{v}_{c}=\bar{v}_{0}-\frac{k C}{\bar{C}+a},
$$

which can be transformed to a straight line by expressing it as:

$$
\frac{C}{\overline{v_{0}}-\overline{v_{c}}}=\frac{C}{k}+\frac{a}{k}
$$

Since $a / k$ is only an additive constant, a straight line is obtained if $C /$ $\left(\bar{v}_{0}-\bar{v}_{\mathrm{c}}\right)$ is plotted vs. $C / k$ (Fig. 4). Of course, the data for the number of spermatozoa $N$ follow the same relationship, with $N$ substituted for $\bar{v}$, since the identity of these relationships was already demonstrated with respect to the Freundlich type of equation.

\section{Effect of Diazine Green on the Velocity Frequency Distribution}

The velocity frequency distributions of the portions to which diazine green had been added were not only shifted to lower values, but their shape was altered, too (Fig. 5). The curves obtained at different concentrations in the range from $2-200$ p.p.m. did not differ significantly among themselves, apart from the mean values, but they were significantly different from the controls. The mean standard deviation $\bar{\sigma}$ (mean biological spread in the

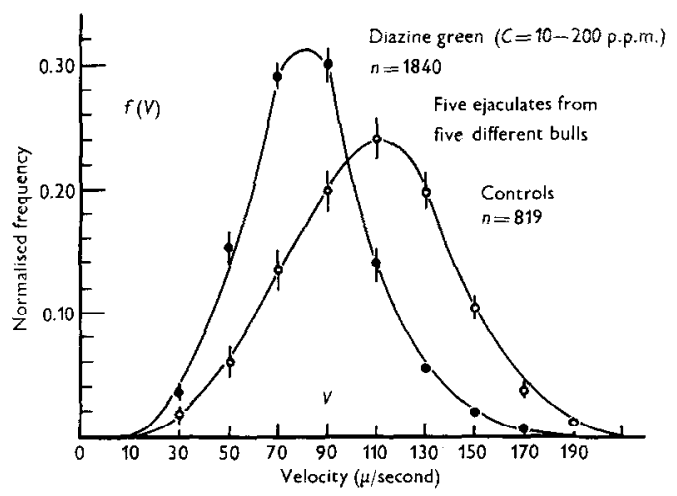

Fig. 5. Normalized velocily frequency distributions of normal bull spermatozoa with diazine green (pooled data from concentrations ranging from 10 up to 200 p.p.m.; these do not differ significantly among themselves) compared with control without vital stain. Pooled data of five ejaculates from five different bulls. 
populations, not error) of the frequency distributions obtained with diazine green present was $25 \pm 0.63$ (S.E.) $\mu /$ sec and that of the controls without dye addition $30 \pm 2.6(S . E$.) $\mu /$ sec. This difference is significant: $0.01<P<$ 0.02 .

The value obtained for the controls is in complete agreement with that of $30 \pm 1.1$ obtained in previous investigations [5].

The type of change of the shape of the frequency distribution curves differs from that observed with acridine orange [2], applied in concentrations ranging from $1: 200,000$ up to $1: 5000$, in all of which only green fluorescence of moving specimens was observed, where the curves became more skew in positive direction with increasing concentration.

\section{Experiments with Varying Light Dosage at Constant Dye Concentration}

With the samples kept in darkness, there was no statistical difference between the semen with diazine green $(20$ p.p.m. $)$ and the control with respect to the rate of decrease of velocity, and the rate of decrease of the number of spermatozoa moving normally; however, the curves were shifted to a lower level. The amount of this difference could be accounted for com-

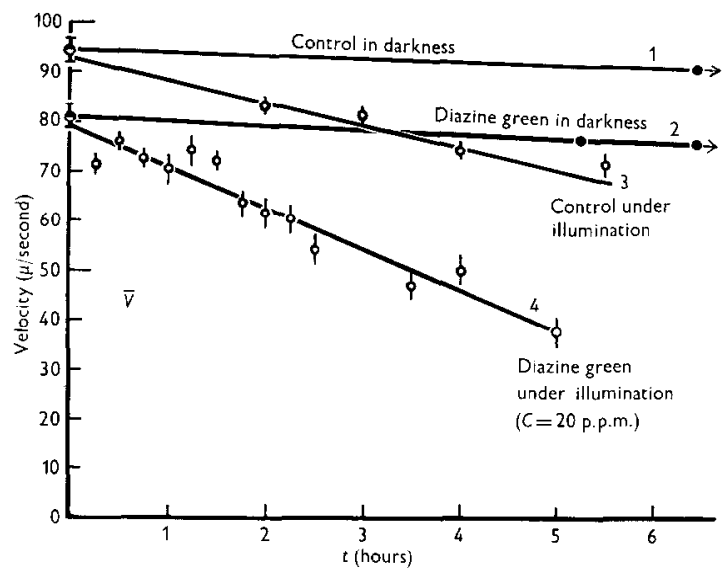

Fig. 6.-Mean velocities of bull spermatozoa as a function of time, with and without diazine green (concentration 20 p.p.m.).

Curve 1. Control without diazine green, kept in darkness.

Curve 2. Sample with diazine green, kept in darkness. No statistical difference in slope; the shift to a lower level can be accounted for completely as a result of photosensitization during the period of measurement.

Curve 3. Control without diazine green, kept under illumination with white light. The slope differs significantly from that of the sample kept in darkness.

Curve 4. Sample with diazine green, kept under illumination, significantly different from curve 3 . 
pletely by the effect of optical sensitization by diazine green during the period of measurement in the dark field microscope with an $8 \mathrm{~V}, 6 \mathrm{~A}$ incandescent lamp as the light source.

In the illuminated samples the mean velocity of spermatozoa with normal movement decreased much more rapidly in the portions to which diazine green had been added than in the controls without dye addition (Fig. 6). The difference in slope between the curves is highly significant $(0.001<P<$ $0.01)$.

\section{DISCUSSION}

The results of the present investigations indicate that diazine green is not toxic to bull spermatozoa in the concentrations employed (up to 200 p.p.m. or $4 \times 10^{-7} M$ ) in the absence of light. Under illumination, however, it acts as a powerful photosensitizer. The transfer of the absorbed photon energy to the mitochondria to which the dye is specifically adsorbed results in a marked deleterious effect on the rate of velocity decrease with time and on the number of surviving specimens. From these results it may be suggested that the adverse effect observed with respect to cells in tissue culture [8] could also be entirely due to photosensitization and not to intrinsic toxicity of the substance in itself. However, the possibility of some toxicity with respect to cells in tissue culture in darkness cannot be excluded completely, since it has been found [7] that acridine orange, which with respect to bull spermatozoa did not show any toxic effect in darkness [2], still produced a definite effect on L-fibroblasts.

Bull spermatozoa are susceptible to light even in the absence of any sensitizing dye in the medium $[3,10]$, owing to a photosensitizer being present as an intrinsic component of the cell $[10]$. This means that every observation requiring visibility (either directly to the eye, or to any apparatus reacting to light impulses) includes automatically and unavoidably the introduction of an uncertainty with respect to the natural condition of the subject and to the effect one wishes to study. This intrinsic uncertainty may be compared to the well-known Heisenberg uncertainty relations in physies and should receive due attention in discussions of observations of cells under conditions widely different from their natural environment.

The data obtained in the experiments with diazine green are in agreement with the conception that the dye does not combine chemically with any cell component of major importance with respect to either movability or livability, but that the underlying mechanism is restricted to adsorption. This can be deduced from the concentration dependency of the effect following not only 
the Freundlich equation, but also the Langmuir adsorption isotherm. Further, the relationship indicates a strict proportionality of the effect with respect to both the decrease of the total number of spermatozoa moving normally and their mean velocity, with the adsorbed number of dye molecules per unit of area of the susceptible surface. This points to a simple optical sensitization effect without interference with or by the photosensitizer already naturally contained in the cell (admixing of a second dye to a photosensitizer may result in either supersensitization--the sum effect exceeds the sum of the individual effects - or anti-sensitization-the sum effect is less than the sum of the individual effects).

Further, from the identity of the effect, including the numerical value of the slope constants, with respect to both the total number of spermatozoa moving normally and their mean velocity, it may be deduced that there is probably but one site of adsorption responsible for decreasing motility and livability. The numerical value of the constants $k$ in the equations (2), (3) and (5) may be regarded as incidental, depending on the actual illumination conditions during the period of recording. The exponent $p / q$ of the Freundlich type of equation may be expected to be a constant, governed by the true adsorption process only; however, the possibility cannot be excluded that it would itself be dependent on the illumination level, which is the reason why this exponent has been expressed in the form $p / q$ instead of by a single constant as would be sufficient for representation of the dye adsorption itself as a function of dye concentration. It must further be emphasized that the present study has been directed only to the detection of fundamental effects and the analysis of their functional relationships, but not to investigating a possible spread in the numerical data between spermatozoa from different cjaculates and between bulls, which would have required much more extensive material.

\section{SUMMARY}

1. The effects of diazine ${ }^{\circledR}$ green (Janus green), a specific vital stain for mitochondria, on the total number of bull spermatozoa moving normally and on their mean velocity and velocity distributions were studied, using the photo-electric equipment described by Rikmenspoel [12] and by Rikmenspoel and Van Herpen [14].

2. In the absence of light diazine green has no measurable effect on living bull spermatozoa in concentrations up to 200 p.p.m. $\left(4 \times 10^{-7} \mathrm{M}\right)$.

3. Lnder illumination, diazine green acts as a powerful photosensitizer, decreasing the total number of normally moving spermatozoa as well as 
their mean swimming velocity. Both effects may be attributed to one single site of attack.

4. The photosensitizing effect is strictly proportional to the number of dye molecules adsorbed per unit of susceptible area. The quantitative relationships are in agreement with a simple adsorptive process, following the empirical Freundlich equation and also the theoretically founded Langmuir adsorption isotherm.

5. The velocity frequency distributions are not only shifted to lower values, but their shape is altered too. The top value of the normalised frequency curves rises and the standard deviation of the distribution is significantly decreased.

The author wishes to express his appreciation to Miss Elisabeth Muilwijk for her excellent and enthusiastic assistance during the course of these investigations.

\section{REFERENCES}

1. Cowину, E. V., Int. Mon. Ant. Physiol. 29, 473 (1914).

2. DUIJN, C. YAN JR., Nature 187, $1006(1960)$.

3. - - ibid. 189, 76 (1961).

4. Proceedings IVth Int. Congress Animal Reprod., The Hague, June 5-9, 1961 (in press).

5. Dujus, C. van, Jr. and Rikmexspoel, R., J. Agric. Sci. 54, 300 (1960).

6. Hill, R. B., JR., Bensch, K. G. and King, D. W., Nature 184, 1429 (1959).

7. - Exptl. Cell Research 21, 106 (1960).

8. I.fwis, W. H. and I.wwis, M. R., Am. .J. Anat. 17, 339 (1915).

9. Michaelis, I., Areh. mikroskop. Anat. 55, 558 (1900).

10. Norman, Ch. and Goldberg, E., Science 130, 624 (1959).

11. Rikmenspoes, R., Experientia 13, 124 (1957).

12. - - Photoclectric and cinematographic measurements of the "motility" of bull sperm cells. Thesis, Utrecht, 1957.

13. Rikmenspoel, R. and Duis, C. vas, JR., Tijdschr. Diergeneesk. 85, 1002 (1960).

14. Rikmenspoel, R. and Herpen, G. va., Phys. Med. Biol. 2, 54 (1957).

15. Seemann, G., $Z$. wiss. Mikroskop. 47, 323 (1930).

16. VanDemark, N. L., Estergreex, V. L., Jk., Schorr, R. and Kuhlmax, D. E., J. Duiry Sci. 42, 1314 (1959). 